\title{
Spatial Variation of Correlation Times for 1D Phase Turbulence
}

\author{
David A. Egolf \\ Department of Physics and the Center for Nonlinear and Complex Systems \\ Duke University,Durham, NC27708-0305; dae@phy.duke.edu \\ Henry S. Greenside \\ Department of Computer Science, Department of Physics, and the Center for Nonlinear and \\ Complex Systems \\ Duke University, Durham, NC27r08-0129; hsg@cs.duke.edu
}

(October 20, 1993)

\begin{abstract}
For one-dimensional phase-turbulent solutions of the KuramotoSivashinsky equation with rigid boundary conditions, we show that there is a substantial variation of the correlation time $\tau_{c}(x)$ with spatial position $x$ in moderately large systems of size $L$. These results suggest that some timeaveraged properties of spatiotemporal chaos do not become homogeneous away from boundaries for large systems and for long times.
\end{abstract}

Typeset using REVTEX 
For spatiotemporal chaotic systems (whose spatial correlation length $\xi$ is smaller than the system size $L[1]$ ), an interesting question arises as to whether time-averaged properties may vary spatially. In a thermodynamic limit of infinite system size and for averages over an infinite time interval, one would expect time-averages to be spatially-independent as a consequence of ergodicity and of rotational and translational invariances of the underlying equations. For experiments or simulations of finite-size, however, spatial variation may arise from boundary conditions, from broken symmetries, or from a combination of both.

Time-averaged inhomogeneous patterns have indeed been found for finite-sized systems both numerically and experimentally. Zaleski [2] and Pumir [3] studied long-time statistical properties of spatiotemporal chaotic solutions of the one-dimensional Kuramoto-Sivashinsky (KS) equation

$$
u_{t}+u_{x x}+u_{x x x x}+u u_{x}=0, \quad \text { for } 0 \leq x \leq L,
$$

with rigid boundary conditions

$$
u=u_{x}=0 .
$$

The time-averaged pattern $p(x)=\langle u(x, t)\rangle$ [2] and simple statistical quantities like the kurtosis [3] (derived from time series measured at a single point in space) were found to vary spatially, but only for spatial intervals small compared to the system size and only near the boundaries. An example is given in Fig. 目, which represents a more thorough time-averaging than that reported in Ref. [2]. We found that the maximum magnitude of the spatial average was independent of the system size $L$ over the range $50 \leq L \leq 2000$, suggesting that the mean spatial pattern is a consequence of the boundary conditions.

More recently, Gluckman et al [4] found quite striking time-averaged two-dimensional spatial patterns in a Faraday crispation experiment for system sizes that were about five times larger than the correlation length $\xi$. Unlike the results found for the KS equation, the time-averaged experimental patterns showed a highly-ordered periodic lattice with the symmetry of the lateral boundaries (square or circular). Similar results have been reported 
by Ning et al for rotating convecting fluid in cells of different symmetry [5]. Although a systematic study of how the magnitude of these patterns decreases with aspect ratio was not made, the results for both numerics and experiments are consistent with the expected behavior that the magnitude of the time-averaged patterns should vanish in the infiniteaspect-ratio limit.

Somewhat related to these investigations of time-averaged patterns are two studies [6,7] concerning whether the fractal dimension $D_{2}$ [8] varies in space. This is a possibility since the fractal dimension is related to the strength and number of active modes, and these can vary spatially for finite-aspect-ratio systems [9]. Sato et al [6] and Ciliberto [7] obtained their results for chaotic convection in rectangular cells of modest-sized aspect ratios, respectively $15 \times 1$ and $4 \times 1$. They measured long time series at many spatial points and analyzed these time series with the Grassberger-Procaccia algorithm [10] to extract $D_{2}(x)$. Sato et al found a strong spatial dependence of Fourier modes but no clear spatial dependence of $D_{2}$. Ciliberto found a stronger spatial dependence of $D_{2}$ in the smaller cell. Both experimental results were not entirely convincing since dimension algorithms based on time series are difficult to apply in a reliable way [11]. Although an unambiguous dependence of fractal dimension on position was not found, Ciliberto did observe a strong spatial variation of the root-mean-square of an observable, with the variation occurring on lengthscales smaller than a roll size.

We note that the above studies concern issues that are somewhat different than those raised by the study of coherent structures in more fully developed turbulent flows [12,13. Coherent structures typically persist only over finite time intervals and long-time averages have not yet been carried out to see how the time-averaged pattern may be related to existing coherent structures.

In this Letter, we look at a different aspect of spatial-variations of time-averages. We investigate the question of whether temporal correlations, as quantified by a correlation time $\tau_{c}$, can vary spatially. This is a rather different question than looking at time-averages of fluctuations since time correlation functions involve comparing information separated at 
time intervals that can be quite large. In the following, we show empirically that there is an unexpected spatial variation of the correlation time for a one-dimensional model of spatiotemporal chaos but only for rigid boundary conditions and only for phase turbulent states [14]. Unlike the numerical [2, 3] and experimental [4,5] results discussed above, the spatial dependence of $\tau(x)$ is strongest in the interior, away from the boundaries. This spatial dependence exists only in the phase-turbulent KS equation, is weakly sensitive to the choice of initial conditions, and disappears with sufficiently long averaging in the more disordered defect-turbulent regime of the CGL equation [15,16].

One implication of these results is that not all features of large-aspect-ratio spatiotemporal chaos become homogeneous away from boundaries, even for systems that are quite large compared to the correlation length (for our calculations, this ratio is about 20). Experimentalists and computational scientists will have to be wary of which point they pick in space to sample information, at least in systems with long-lived temporal correlations as is the case for phase turbulence.

We obtained our results by numerical integrations over times $T$ as large as $10^{6}$ time units for Eqs. (1]) and (2), and for the closely related but more general complex-Ginzburg-Landau (CGL) equation [14]

$$
\partial_{t} A(x, t)=A+\left(1+i c_{1}\right) \partial_{x}^{2} A-\left(1-i c_{3}\right)|A|^{2} A,
$$

with Dirichlet boundary conditions

$$
A(0, t)=A(L, t)=0 .
$$

The CGL field $A(x, t)$ is complex-valued and the parameters $c_{1}$ and $c_{3}$ are real-valued. The KS equation can be derived [14] from the CGL equation in the Benjamin-Feir unstable limit $c_{1} c_{3} \rightarrow 1$. For fixed $c_{1} \geq 1.8$, there is an apparent transition from phase turbulence, for which the field magnitude $|A|$ is bounded away from zero for all time, to defect turbulence, in which isolated space-time defects of zero field $A$ value occur [16]. It is not yet known whether the two phases are distinct in the thermodynamic limit of an infinitely large system, 
e.g., phase turbulence may simply be a defect turbulence with an unobservably low but finite density of space-time defects. Unpublished results of ours, extending Shraiman et al to much larger system sizes, suggest that the phases are in fact distinct.

We integrated Eqs. (11) and (2) and Eqs. (3) and (4) with second-order-accurate finitedifference codes with time-splitting of the linear and nonlinear operators [17]. At each of many coordinates $x$ on a regular spatial mesh spanning the interval $[0, L]$, we calculated a correlation time $\tau_{c}(x)$ from the $x$-dependent time correlation function

$$
C(\tau ; x)=\langle(u(t+\tau, x)-\bar{u}(x))(u(t, x)-\bar{u}(x))\rangle_{t},
$$

where $\bar{u}(x)$ is the function representing the time averaged mean at each point in space and where the brackets indicate time averaging over information at position $x$. This function was found to decay approximately exponentially for small times. We used the root-mean-square width of $C(\tau ; x)$ up to the first zero crossing to determine the correlation time $\tau$. Our results (especially Fig. 3) are not sensitive to our method of extracting $\tau$, e.g., we found similar results when estimating $\tau$ by the first-zero-crossing of $C(\tau)$ or by the integral time scale 18 .

We note that the long-time long-wavelength dynamics of the KS-equation and of the phase-turbulent regime of the CGL equation is believed to be described by the Kardar-ParisiZhang (KPZ) equation [19 22] which describes the roughening transition of an interface. If this asymptotic description is correct, the time correlation function in one spatial dimension does not decay exponentially with a single well-defined time scale, but instead decays as a stretched exponential of the form $\exp \left(-B t^{2 \beta}\right)$ with $\beta=1 / 3$. We ignore this subtlety in what follows since, as shown in Fig. 2, there is a substantial decay of correlations over a finite time interval and the system sizes are small compared to the lengthscale over which the KPZ description becomes valid [20].

We also ignore a possibly serious issue concerning whether our numerical results are statistically stationary so that all transients have decayed sufficiently. Naively, one would hope that an integration time $T$ of order $10^{6}$ that is many orders of magnitude greater than the correlation time $\tau_{c} \leq 40$ would be sufficient for transients to decay. However, some calcula- 
tions by Shraiman 23] suggest that KS phase turbulence with periodic boundary conditions is transient, with a decay time that grows exponentially with system size $L$. (It is not known if this conclusion generalizes to the boundary conditions Eq. (2) although numerical calculations suggest that this is unlikely). The possibility of extremely long transient times has also been argued from numerical studies of coupled map lattices [24,25]. We do not know of any quantitative method for distinguishing such long-lived transients from nontransient chaos, nor do we know whether there are observable dynamical implications of averaging over such a transient rather than over an attractor. We present the results below with the hope that, if they are transient, the slow decay times lead to an ergodic average that is approximately correct.

Our results are summarized in Figs. 2 through 5 . Fig. 2 shows parts of the temporal and spatial correlation functions averaged over all space and time, from which correlation times and lengths were calculated for the KS equation with rigid boundary conditions. We estimate a value of $\tau=2$ units and of $\xi=7$ units respectively and choose integration times and system sizes much larger than these values in what follows.

Fig. 36(a) shows the central result of this Letter. The correlation time of the KS equation, as measured by the root-mean-square width of the temporal correlation function for rigid boundary conditions, varies substantially with spatial position. The magnitude of this spatial variation is quite large, with some peaks being as large as five times the average background value of about 8 . The peaks show structure that varies substantially over length-

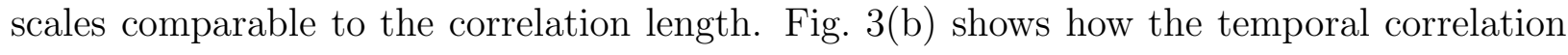
function itself varies over a small spatial interval $25 \leq x \leq 30$ of the system. The variation in correlation times between $x=25$ and $x=30$ is caused by the presence or absence of the small dip in the correlation function around $\tau=12$. The variations in the correlation function at large $\tau$ remain about the same magnitude out to the largest values of $\tau$ that we tested. When averaged over space, these variations disappear as shown in Fig. 2(a).

From similar numerical calculations, we have found empirically that the positions of the peaks in Fig. 通(a) do not change with the length of the integration time $T$, with different 
initial conditions or for changes in the numerical time resolution. However, the relative heights and widths of the peaks do change under these conditions.

To obtain more insight about the origin of these peaks, we have made similar calculations for the one-dimensional complex Ginzburg-Landau equation, Eq. (3), which has a phaseturbulent regime similar to the KS equation but also a more strongly disordered defectturbulent regime for which temporal correlations decay much more rapidly [16,26]. The spatial variation of Fig. 3(a) is not seen for defect-turbulent states with rigid boundary conditions, Eq. (凹), as shown in Fig. 世. Fig. 4(a) shows the time averaged mean of the amplitude of the field $A$ as a function of position while Fig. 4(b) shows how the correlation time varies with position. In both cases, peaks are observed near the boundaries while the bulk variations are small and smooth. The asymmetry in the heights of the outermost large peaks may be a consequence of some small initial asymmetry in the initial conditions. (We have not studied rigid boundary condition solutions in the phase-turbulent regime of the CGL equation, which presumably would give results similar to Fig. 3.) We deduce that the spatial variation of the correlation time is a consequence of the long-lived time correlations present in phase turbulence.

We have also tested the importance of rigid boundary conditions by numerically integrating the 1d CGL equation with periodic boundary conditions in the phase-turbulent regime. In this case, we find that there is no spatial variation of the correlation time as shown in Fig. 5. Essentially identical results are found for the KS equation with periodic boundary conditions with the difference that one finds substantial spatial variations for short integration times that slowly decay away in magnitude for longer integration times. These results confirm our earlier summary of previous numerical and laboratory experiments [2] 5], that average spatial patterns are driven by the presence of boundary conditions that break the translational symmetry.

In conclusion, we have looked at a variation of the idea that time-average spatiotemporal chaotic patterns can have interesting mean structure. By numerical simulations on the KS and CGL equations in one-space dimension, we have found that the correlation time $\tau_{c}$ can 
vary substantially in space when there are long-lived temporal correlations (phase turbulence) and for boundary conditions that break translational invariance. For dynamical states with more rapidly decaying correlation functions (defect turbulence) or for periodic boundary conditions, there is no interesting spatial variation of $\tau_{c}$ provided that one integrates sufficiently long in time. It would be interesting to explore these issue further in laboratory experiments on fluids and flames.

We thank Geoff Grinstein for several useful discussions. This work was supported by National Science Foundation Grant ASC-8820327, by a fellowship from the Office of Naval Research, and by allotments of CRAY CPU time at the North Carolina Supercomputing Center and at the National Center for Supercomputing Applications. 


\section{REFERENCES}

[1] M. C. Cross and P. C. Hohenberg, Rev. Mod. Phys. 65, 851 (1993).

[2] S. Zaleski and P. Lallemand, J. Physique Lett. 46, L793 (1985).

[3] A. Pumir, J. Physique 46, 511 (1985).

[4] B. J. Gluckman, P. Marcq, J. Bridger, and J. P. Gollub, Phys. Rev. Lett. 71, 2034 (1993).

[5] L. Ning, Y. Hu, R. E. Ecke, and G. Ahlers, Phys. Rev. Lett. 71, 2216 (1993).

[6] S. Sato, M. Sano, and Y. Sawada, Phys. Rev. A 37, 1679 (1988).

[7] S. Ciliberto, Europhys. Lett. 4, 685 (1987).

[8] J.-P. Eckmann and D. Ruelle, Rev. Mod. Phys. 57, 617 (1985).

[9] E. N. Lorenz, Nature 353, 241 (1991).

[10] P. Grassberger and I. Procaccia, Physica D9, 189 (1983).

[11] D. Ruelle, Proc. R. Soc. London A427, 241 (1990).

[12] B. J. Cantwell, Ann. Rev. Fluid Mech. 13, 457 (1981).

[13] A. K. M. F. Hussain, J. Fluid. Mech 173, 303 (1986).

[14] Y. Kuramoto, Chemical Oscillations, Waves, and Turbulence (Springer-Verlag, New York, 1984).

[15] H. Sakaguchi, Prog. Theor. Phys. 84, 792 (1990).

[16] B. I. Shraiman et al., Physica D 57, 241 (1992).

[17] P. Manneville, Dissipative Structures and Weak Turbulence, Perspectives In Physics (Academic Press, New York, 1990). 
[18] H. Tennekes and J. L. Lumley, A First Course in Turbulence (The MIT Press, Cambridge, Mass., 1972).

[19] M. Kardar, G. Parisi, and Y.-C. Zhang, Phys. Rev. Lett. 56, 889 (1986).

[20] K. Sneppen et al., Phys. Rev. A 46, 7351 (1992).

[21] I. Procaccia et al., Phys. Rev. A 46, 3230 (1992).

[22] G. Grinstein and C. Jayaprakash (unpublished).

[23] B. I. Shraiman, Phys. Rev. Lett. 57, 325 (1986).

[24] J. P. Crutchfield and K. Kaneko, Phys. Rev. Lett. 60, 2715 (1988).

[25] S. V. Ershov and A. B. Potapov, Phys. Letts. A 167, 60 (1992).

[26] D. A. Egolf and H. S. Greenside, submitted to Nature. Available as a preprint from the Los Alamos archive, number patt-sol/9307010 (unpublished). 


\section{FIGURES}

FIG. 1. Spatial dependence of (a) the time-averaged mean-value $\langle u\rangle$ and of (b) the time-averaged square-deviation $\left\langle(u-\langle u\rangle)^{2}\right\rangle$ for solutions of the one-dimensional $\mathrm{Ku}$ ramoto-Sivashinsky equation Eq. (1/) with rigid boundary conditions Eq. (2) on an interval of size $L=100$. The temporal resolution was $\triangle t=0.1$, the spatial resolution was $\triangle x=0.5$, and the total integration time was $T=10^{6}$ time units. For initial data, we used uniformly distributed random numbers in the interval $[-0.03,0.03]$.

FIG. 2. (a) Plot of the temporal correlation function Eq. (5) averaged over all space and time for a numerical solution of the KS equation, Eqs. (11) and (2). There is a substantial decay of correlation over a time scale $\tau_{c}=2$. The numerical parameters are the same as those given in Fig. 1. (b) Space- and time-averaged spatial correlation func-

tion $S(y)=\langle(u(t, x+y)-\bar{u}(t))(u(t, x)-\bar{u}(t))\rangle_{x, t}$ plotted over the interval $0 \leq x \leq 50$ for a system of size $L=100$. There is an oscillatory exponential decay of correlations, defining a spatial correlation length $\xi=7$ that is small compared to the system size $L$.

FIG. 3. (a) Plot of the root-mean-square (rms) width of the correlation function $C(\tau)$ as a function of position $x$, for the same parameters used in Figs 1 1 and 2. A substantial spatial dependence of $\tau_{c}$ is found. (b) Details of the variations of $C(\tau)$ over the spatial interval $25 \leq x \leq 30$. The initial decay of the correlation function has not been plotted to allow smaller features of the function to be seen on the same scale.

FIG. 4. (a) The time-averaged mean value and (b) the rms width of the time correlation function $C(\tau)$ of the amplitude of the field in the CGL equation with rigid boundary conditions, Eqs. (3) and (4). Results are presented for the parameter values $c_{1}=3.5$ and $c_{3}=1.5$, which is in the defect-turbulent regime [16]. Spatial variations are found only near the boundaries. The temporal resolution was $\triangle t=0.05$, the spatial resolution was $\triangle x=0.25$, and the total integration time was $T=4 \times 10^{6}$ time units. 
FIG. 5. Plot of the rms width of the time correlation function $C(\tau)$ for periodic boundary conditions of the $1 \mathrm{~d}$ CGL equation for parameter values $(c 1, c 3)=(3.5,0.7)$. The numerical parameters were $L=100,256$ Fourier modes, $\Delta t=0.2$, and $T=10^{6}$ time units. After a sufficiently long time-averaging, the correlation time is no longer varying spatially. 

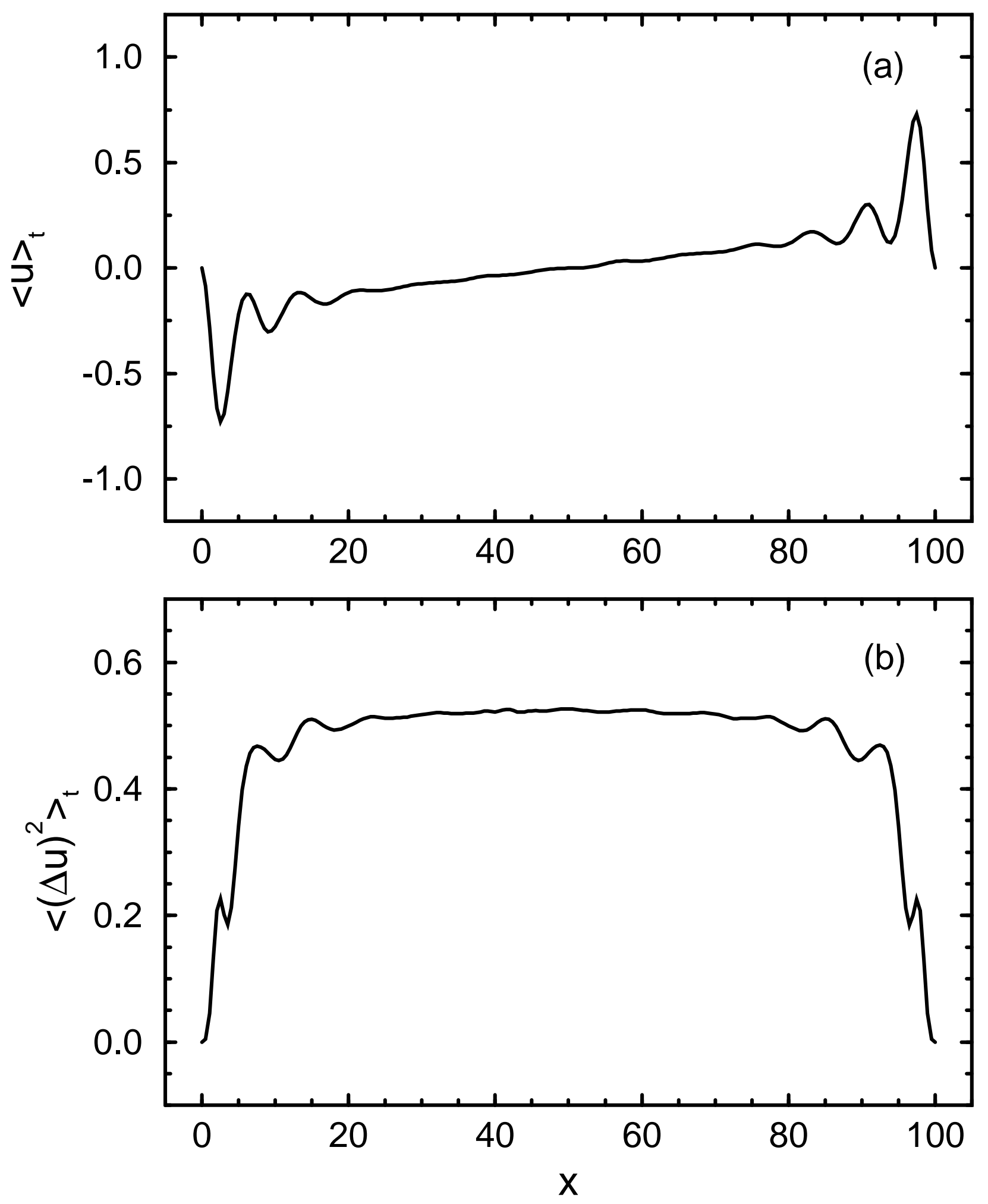

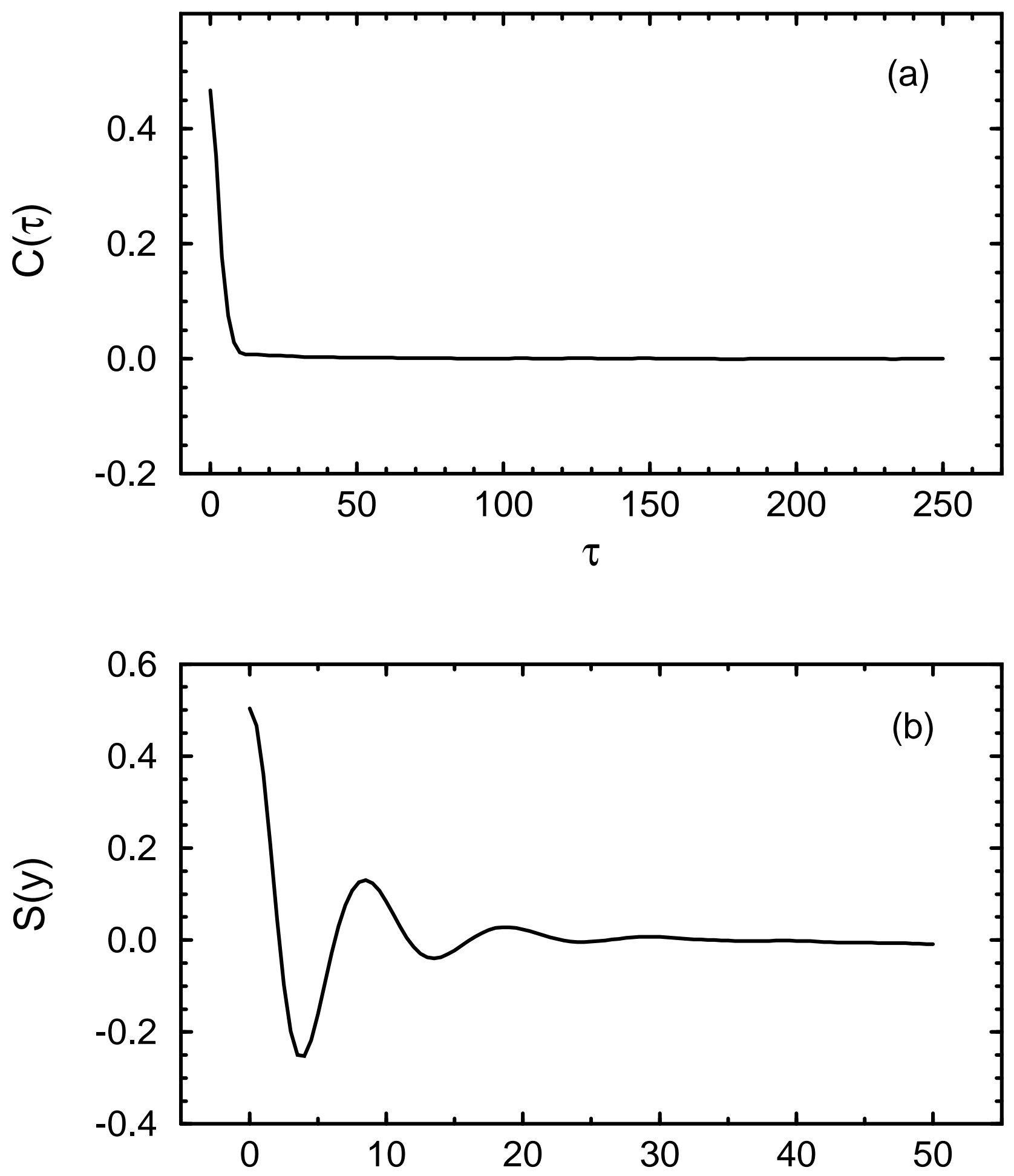

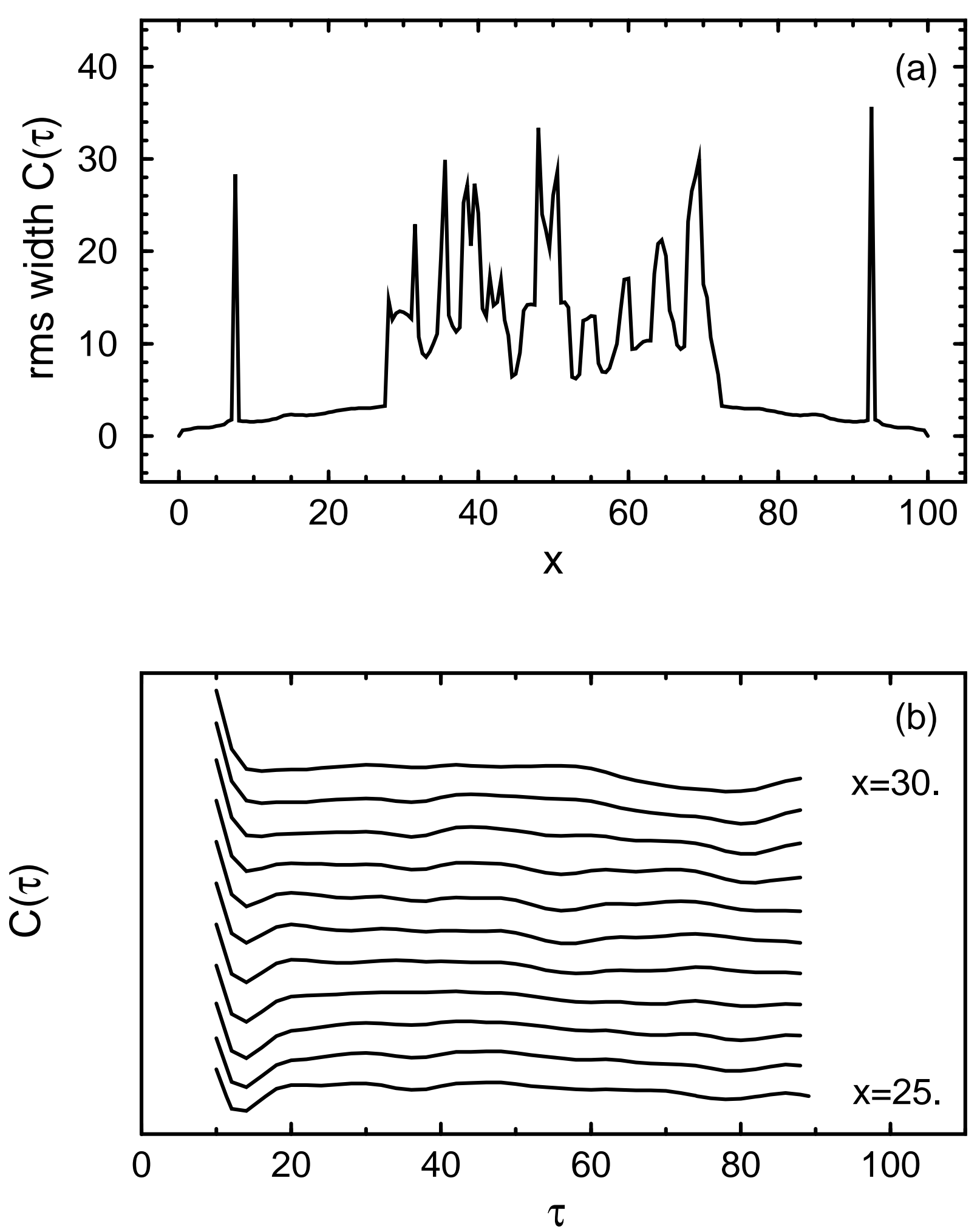

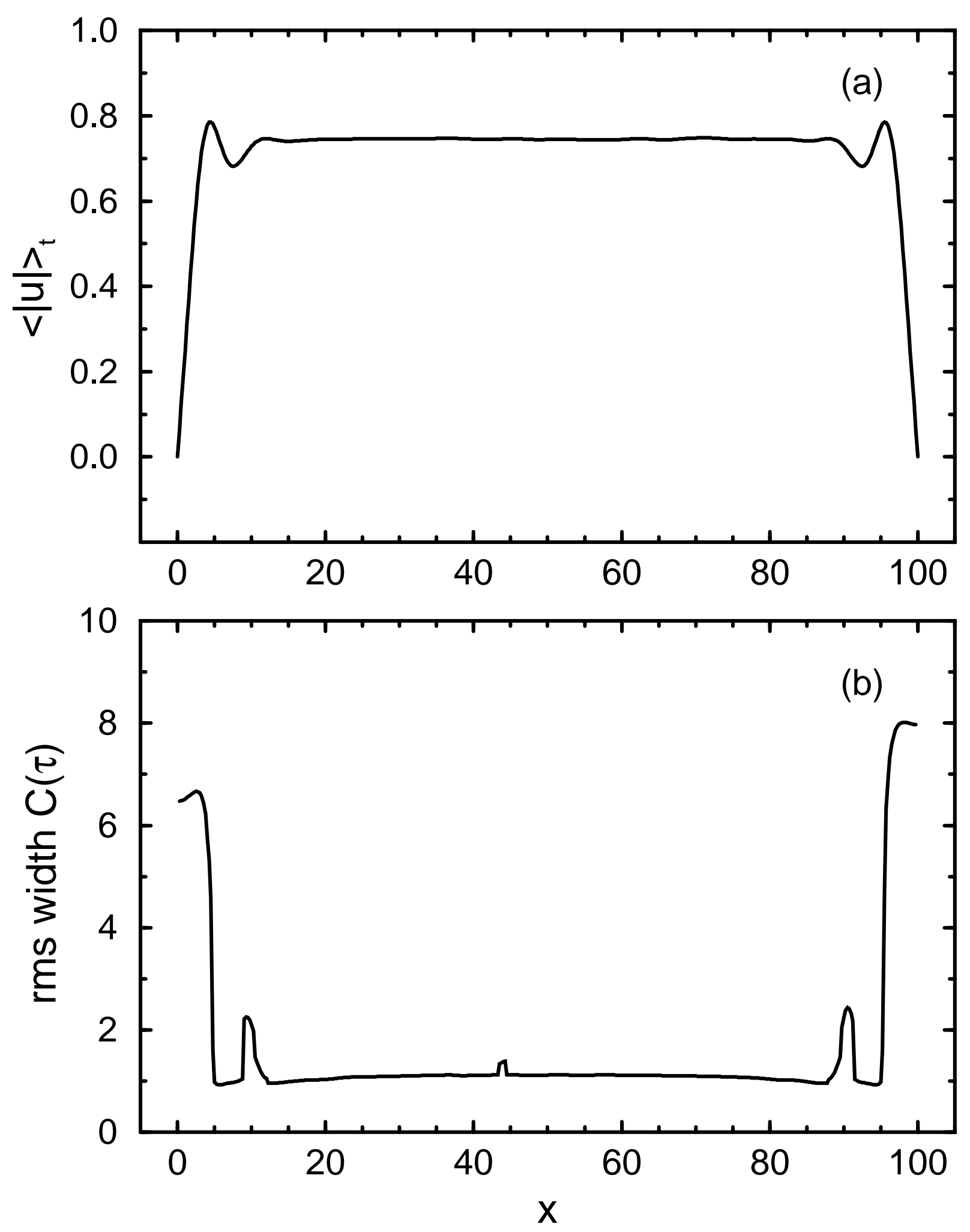


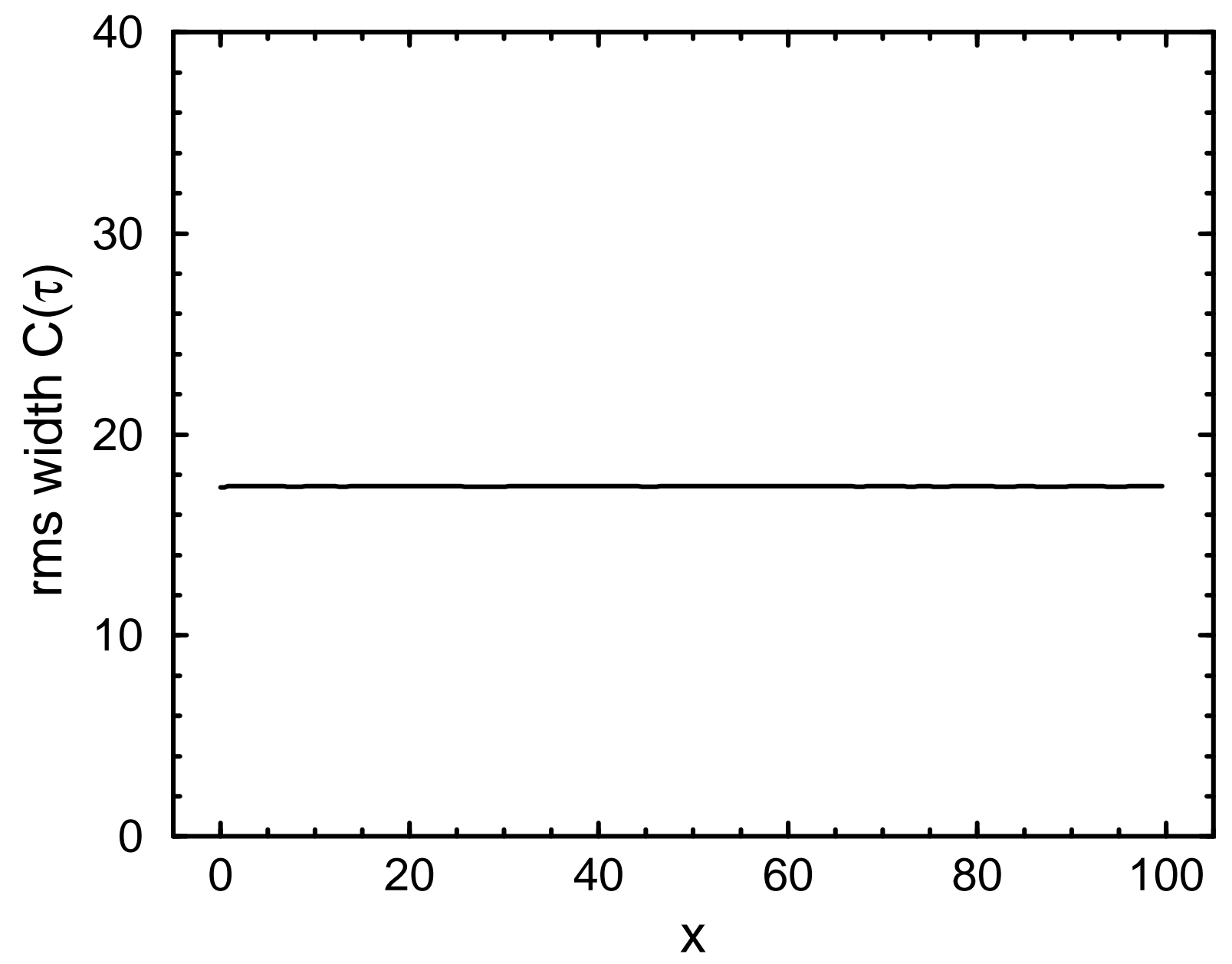

